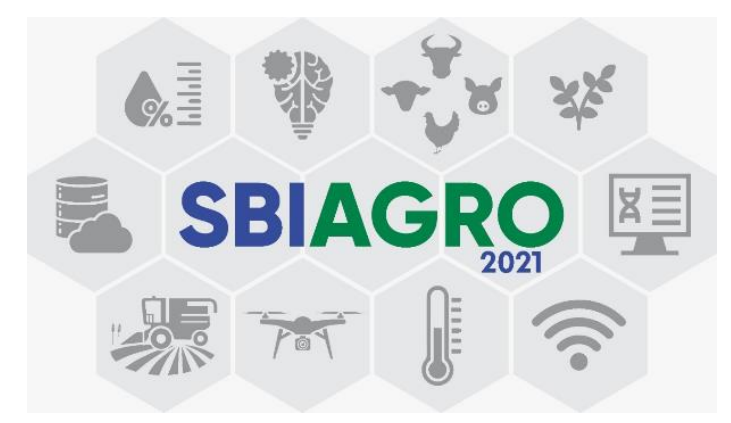

\title{
Monitoramento de plantas em casas de vegetação para assimilação de dados
}

\author{
Monique Pires Gravina de Oliveira ${ }^{1}$, Luiz Henrique Antunes Rodrigues ${ }^{1}$ \\ ${ }^{1}$ Faculdade de Engenharia Agrícola - Universidade Estadual de Campinas \\ (Unicamp) \\ moniquepgoliveira@gmail.com, lique@unicamp.br
}

\begin{abstract}
Data in agriculture is being produced by new sources with new roles and great potential. They may be captured on the field using the internet of things or in plant factories and they may be used on digital twins, which aim at connecting observations with a model of the system. This connection may happen using data assimilation and, particularly in controlled environments, plants may be more continuously monitored, allowing for more data to be available. In this work, we performed data assimilation in a tomato model using data from cameras and load cells, observing these sources provide good estimates of aboveground biomass and that assimilation improves estimates obtained by the non-calibrated Reduced-State Tomgro model.
\end{abstract}

Resumo. Mais dados têm sido gerados em aplicações agrícolas por novas fontes e com grande potencial de uso. Obtidos no campo ou em fazendas verticais, eles podem ser usados, por exemplo, em gêmeos digitais, que visam conectar observações a um modelo do sistema. Essa conexão pode ocorrer por assimilação de dados e em ambientes protegidos, em que as plantas podem ser monitoradas mais intensamente, mais dados estariam disponiveis. Neste trabalho, realizamos assimilação em um modelo de tomateiro usando dados coletados por câmeras e por células de carga, observando que essas fontes fornecem boas estimativas de biomassa da parte aérea e que a técnica melhora as estimativas obtidas pelo modelo Tomgro Reduzido sem calibração.

\section{Introdução}

O uso de dados em modelagem tem sido tradicionalmente associado ao começo do ciclo de modelagem, no seu desenvolvimento, ou ao fim, para calibração e avaliação dos modelos. Existem, no entanto, novas expectativas para seu uso que são baseadas em sua abundância e qualidade. [Keating and Thorburn 2018] mencionaram uma nova forma de se relacionar com dados que expandiria esse papel por meio de, por exemplo, 
modelagem inversa e fusão de modelo e dados, incluindo novos papéis para sensoriamento remoto e proximal em sua inicialização e calibração.

A assimilação de dados é um método amplamente utilizado em hidrologia e meteorologia e consiste em combinar observações aos estados estimados pelos modelos, levando em consideração a incerteza que existe nas estimativas dos modelos e nas observações [Pellenq e Boulet 2004]. No caso da modelagem de culturas agrícolas, a assimilação de dados tem sido usada com imagens de sensoriamento remoto para estimar variáveis de estado relacionadas ao dossel ou a propriedades do solo, visando obter melhores estimativas de produtividade, índice de área foliar e umidade do solo [Dorigo et al. 2007; Jin et al. 2018].

Revisões recentes discutem as diferentes abordagens para realizar a assimilação e suas limitações [Huang et al. 2019; Jin et al. 2018]. Elas apresentam os aspectos teóricos das técnicas bem como os diversos algoritmos que têm sido utilizados, incluindo diferentes contextos e objetivos. Dentre as limitações elencadas pelos autores, frequentemente são apontadas questões relacionadas ao uso em grandes áreas, visto que assimilação de dados tem tido sua aplicação frequentemente limitada às resoluções temporais e espaciais de satélites. Trabalhos recentes exploraram outras fontes de dados, como veículos aéreos não-tripulados (VANTs) e imagens digitais. [Yu et al. 2020] se valeram dessas outras fontes para melhorar as estimativas de produtividade por meio da assimilação da altura da cana-de-açúcar. [Linker and Ioslovich 2017] usaram imagens digitais do dossel para estimar a cobertura do dossel a ser assimilada no modelo Aquacrop, bem como dados de análises destrutivas como observações de biomassa. Dados de análises destrutivas também foram usados por [Ruíz-García et al. 2014] com alfaces e o modelo Nicolet.

Dado que é possível monitorar mais intensamente o crescimento da vegetação em cultivo protegido, também é possível, com maiores resoluções temporais e espaciais, realizar a assimilação com observações coletadas automaticamente em modelos de crescimento de hortaliças e outras culturas normalmente cultivadas em estufas agrícolas e outros ambientes protegidos. Medições automáticas relacionadas à planta em ambientes protegidos tem sido aplicada para obtenção de diferentes informações. $\mathrm{O}$ monitoramento do crescimento da planta por meio de células de carga foi registrado na Holanda por [De Koning and Bakker 1992] e sugerido por trabalhos como [Helmer et al. 2005; Lee and Son 2019] e em [Chen et al. 2016], para fazendas verticais. Outras aplicações possíveis estão relacionadas à determinação da demanda de água pela planta [De Graaf et al. 2004] e em "gêmeos digitais" [Verdouw et al. 2021]. A assimilação dessas observações poderia aprimorar estratégias de modelagem de [Marcelis et al. 2000], com informações de feedback por sensores, ou complementar as estratégias que têm explorado principalmente métodos de aprendizado de máquina para lidar com dados em tempo real [Gong et al. 2021; Hemming et al. 2019, 2020]. E como a variabilidade da produção em estufas pode ser detectada até mesmo por modelos [Bojacá et al. 2009], esta abordagem pode melhorar a avaliação da produção pelos produtores.

Como a assimilação de dados com dados de diferentes fontes pode se mostrar útil neste ambiente, valendo-se do monitoramento intenso e de modelos de crescimento vegetal, e como o tomate é uma cultura mais estudada e relevante para a economia, é possível que técnicas de assimilação de dados sejam capazes de melhorar as estimativas 
obtidas por um modelo de crescimento de tomateiros mesmo sem calibração. O objetivo deste trabalho foi então investigar, com os dados obtidos em um ambiente de produção, se uma técnica de assimilação seria capaz de aprimorar as estimativas de biomassa da parte aérea realizadas pelo modelo Tomgro Reduzido (Reduced State Tomgro) [Jones et al. 1999].

\section{Material e Métodos}

\section{Modelo de crescimento}

O modelo Tomgro Reduzido (RT) é um modelo que resume as principais características do modelo TOMGRO e foi desenvolvido para utilização em sistemas de controle de cultivos protegidos. O modelo possui apenas cinco variáveis de estado - número de nós, índice de área foliar, biomassa seca da parte aérea, biomassa seca de frutos e biomassa seca de frutos maduros - e foi desenvolvido visando o controle do ambiente. É um modelo simples que não inclui crescimento radicular ou irrigação, e essa simplicidade pode ajudar nesta primeira abordagem.

Com base em dados horários de temperatura e radiação solar, o modelo quantifica o crescimento e o desenvolvimento do tomateiro quando a água e os nutrientes não são limitantes. Seu desenvolvimento é indicado por meio do número de nós e o seu crescimento, por meio dos demais estados. $\mathrm{O}$ cálculo da biomassa seca da parte aérea se dá pela diferença entre a fração de carboidratos destinada à parte área da planta e a fração removida por senescência das folhas ou desfolha. A fração de carboidratos, por sua vez, é calculada como a diferença entre a fotossíntese e a respiração, multiplicada por um fator de que caracteriza a eficiência de conversão dos fotoassimilados em carboidratos, e por um coeficiente que indica qual a proporção da massa que não é destinada às raízes. Mais informações sobre variáveis e parâmetros podem ser encontradas em [Jones et al. 1999].

\section{Coleta de dados}

Foram realizados três ciclos de crescimento de minitomates, conduzidos em estufas agrícolas de pesquisa na Faculdade de Engenharia Agrícola da Universidade Estadual de Campinas, localizada no município de Campinas, SP. O Ciclo 1 teve início em julho de 2019 e término em outubro de 2019, tendo sido utilizada a cultivar Fercan/Milla, o Ciclo 2 foi iniciado em novembro de 2020 e concluído em fevereiro de 2021, com a cultivar Feltrin/Carolina e o Ciclo 3 foi realizado entre março de 2021 e junho de 2021, com a cultivar Seminis/DRC-564.

Os dados ambientais medidos referem-se à temperatura do ar e à radiação fotossinteticamente ativa (RFA). A temperatura foi determinada por transdutores SHT75 protegidos por cápsulas porosas que, por sua vez, eram protegidas por tubos de policloreto de vinila revestidos com folha de alumínio. Os tubos incluíam ventiladores a jusante. Os sensores foram instalados em plataforma de hardware para redes de sensores sem fio (Radiuino BE900), com backup diário. Para RFA, foram utilizados sensores Licor LI-190SA com um datalogger Licor LI-1400.

As plantas foram caracterizadas por análises destrutivas e por observações não destrutivas. Os dados não-destrutivos referem-se às fotos tiradas lateralmente, por módulos fixos de câmera Raspberry Pi v2, conectados a um computador Raspberry Pi 
Zero. Também se referem ao monitoramento contínuo - a cada minuto - de peso utilizando transdutores de força HBM S2M com força nominal de $10 \mathrm{~N}$ (precisão de 0,02\%) e armazenados em um datalogger PMX WGX002, cartão de medida PX455. Nos Ciclos 02 e 03, o tempo total de irrigação foi definido como aquele que não permitiria déficit ao final do dia, para minimizar as flutuações de massa de um dia para o seguinte, causadas pela variação nas irrigações.

Os dados destrutivos referem-se à caracterização do peso seco da planta. Em intervalos de uma a três semanas, três plantas eram retiradas da casa de vegetação. Folhas, haste e frutos maduros e verdes foram separados para pesagem. O material vegetal foi pesado antes e depois da secagem por quatro dias ou até que o peso constante fosse atingido.

Antes da remoção, essas plantas foram fotografadas lateralmente com uma câmera de smartphone. Todas as fotos incluíram referências de tamanho. A anotação dos órgãos das plantas nas imagens foi feita manualmente, por meio do software GIMP $^{1}$. Foram marcadas apenas as áreas em que havia confiança de que o órgão correspondia à planta correta, o que fazia com que se houvesse incerteza ou oclusão a área não fosse marcada. Os órgãos foram coloridos de forma diferente, sendo então detectados por um script em Python. A área correspondente foi calculada usando a referência.

Os três ciclos apresentaram desenvolvimentos distintos, que são explorados neste trabalho: o primeiro pode ser caracterizado por baixa irrigação, submetendo as plantas a déficit hídrico, o segundo, por excesso de fertilização com nitrogênio e ataque do ácaro do bronzeamento ao final do ciclo, e o terceiro ciclo foi conduzido de forma mais próxima ao potencial.

Os dados das análises destrutivas foram usados para calibrar o modelo de Tomgro Reduzido. A calibração seguiu a abordagem de minimizar o erro quadrado relativo. Dado que havia três ciclos diferentes, cada um com condições diferentes, as simulações denominadas calibradas usaram dados do respectivo ciclo. Simulações não calibradas usaram dados da calibração original do modelo.

\section{Modelos de observação}

Dados obtidos por formas não-destrutivas para as plantas submetidas às análises destrutivas, bem como os resultados destas análises foram usados para criar modelos de observação. Nossa estratégia de modelagem focou na obtenção de relações empíricas simples e o método dos mínimos quadrados generalizado foi usado para lidar com a heterocedasticidade e a correlação entre os resíduos. Para evitar vazamento de dados, apesar das diferentes condições de crescimento, os dados do ciclo não foram usados para obter a relação usada naquele ciclo. Todos os valores obtidos por planta foram multiplicados pela densidade de plantas para compatibilizar as unidades com os valores estimados pelo modelo para o estado.

Usamos a área e a altura extraídas de imagens laterais para inferir a biomassa acima do solo e o peso total, conforme determinado pelo sistema de pesagem para

\footnotetext{
${ }^{1}$ GNU Image Manipulation Program
} 
inferir a biomassa acima do solo. Nesse caso, realizamos uma conversão que excluiria as raízes, dependendo do estágio de desenvolvimento, e que converteria de biomassa fresca em seca.

\section{Assimilação de dados}

Foi usada a técnica Unscented Kalman Filter (UKF), que requer especificações de incerteza que foram atribuídas como a seguir. A incerteza nos estados iniciais foi determinada como a variância das amostras analisadas no dia do transplantio. A incerteza no modelo para o UKF foi determinada como a raiz do erro quadrático médio calculado pela diferença entre as observações no ciclo e o valor simulado do modelo não calibrado. Embora estejamos cientes de que isso corresponde a vazamento de dados, acreditamos que essa foi a melhor maneira de fornecer uma estimativa para este parâmetro. A variância nas medições foi determinada como o erro padrão residual para o modelo de observação no ciclo. Acreditamos que esta seja uma forma de incorporar erros de medição e erros na representação do estado por meio dessa abordagem. Neste trabalho, a variável de estado estimada foi a biomassa seca da parte aérea.

Uma vez que nosso objetivo era determinar o quanto as estimativas do modelo não calibrado poderiam ser melhoradas assimilando observações de diferentes fontes, avaliamos nossa abordagem comparando as séries não calibradas, calibradas e filtradas.

\section{Resultados e discussão}

\section{Modelos de observação}

Na Figura 1, mostramos as correlações entre os pares de variáveis que deram base aos modelos de observação que obtivemos. É possível notar também as diferenças entre os ciclos. Notam-se a menor amplitude da biomassa total no Ciclo 1, decorrente dos períodos de estresse hídrico, e os maiores valores de biomassa aérea no Ciclo 3, provavelmente ligados ao menor estresse das condições de cultivo. Nos dois primeiros ciclos, ainda que a biomassa total produzida pudesse ser equivalente à do Ciclo 3 , o estresse poderia levar uma parte maior dos assimilados produzidos a serem destinados à raiz.

A figura mostra que as três fontes utilizadas fornecem abordagens razoáveis para estimativa de biomassa. As menores correlações se referem ao uso da altura para estimativa da biomassa no Ciclo 1, em que apesar da altura máxima ter sido atingida, houve pouca produção de folhas e frutos, diminuindo a massa aérea, e ao uso da área de folha nas imagens para caracterização da biomassa no Ciclo 2, em que houve produção excessiva de folhas, inclusive em detrimento da produção de frutos. Pode-se perceber também que, exceto para a altura, as observações estão concentradas nos valores mais baixos, e se por um lado, isso distorceu a distribuição das observações e pode ter destacado observações não informativas, ao mesmo tempo, se a maior parte do ciclo é representada por esses valores baixos, é razoável usá-los para obter os modelos. Uma anotação automática de imagens permitiria monitorar mais plantas e tornar os resultados mais robustos e baseados em mais observações. 


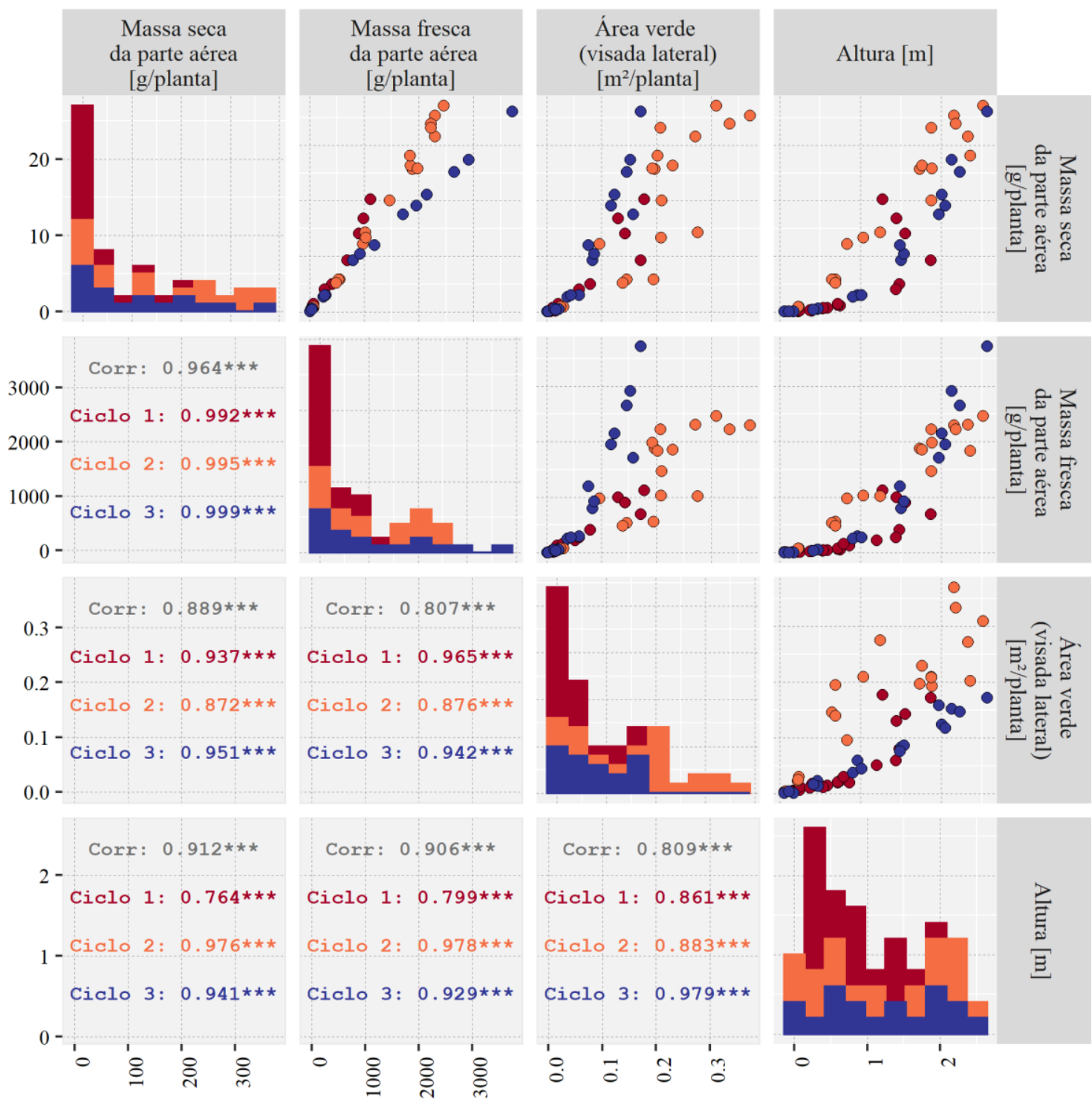

Figura 1. Os gráficos da parte superior mostram os gráficos de dispersão pareados comparando a biomassa da parte aérea às medidas diretas $e$ indiretas obtidas em cada ciclo. Os gráficos da parte inferior apresentam a correlação geral e as específicas para cada ciclo e a diagonal mostra a distribuição dos dados, colorida de acordo com o ciclo de cultivo.

\section{Assimilação de dados}

Nossos experimentos levaram a resultados diferentes com relação ao aprimoramento das estimativas (Figura 2). Frequentemente, o UKF levou a melhorias nas estimativas ao longo do ciclo em comparação ao modelo não calibrado, incluindo resultados tão bons quanto os obtidos pela calibração. Entretanto, esse desempenho variou dependendo da fonte de dados utilizada.

O uso da altura permitiu o aumento das baixas estimativas realizadas pelo RT nos três ciclos. No Ciclo 1, os valores obtidos foram superiores aos das demais plantas no ambiente, enquanto nos Ciclos 2 e 3, embora os valores tenham sido compatíveis aos das demais no fim do ciclo, foram obtidos valores negativos para a massa na fase inicial do crescimento. Isso ocorreu porque a relação entre a biomassa e a altura foi sido tratada 
como linear quando, na verdade, as observações do início do crescimento não têm esse comportamento. Já o uso das áreas verdes identificadas nas imagens também permitiu aumento das estimativas do modelo para o Ciclo 2, tornando-as bem próximas aos valores obtidos pelo modelo calibrado, mas não afetou grandemente o Ciclo 3 . Neste caso, o modelo de observação obtido para o ciclo relacionou as áreas observadas a valores muito baixos de biomassa. Deste modo, não houve muitas diferenças entre as estimativas e as observações, o que não promoveu correções dos valores estimados. Mais ciclos de cultivo permitiriam a obtenção de modelos mais robustos, que melhor representassem as relações entre as observações e os estados. Por fim, a massa do sistema de pesagem ajustou quase perfeitamente o Ciclo 2 e melhorou as estimativas do Ciclo 3, ainda que subestimando o valor final. Para o Ciclo 1, as estimativas foram muito comprometidas em razão do erro na medição causado por flutuação da água durante o crescimento.

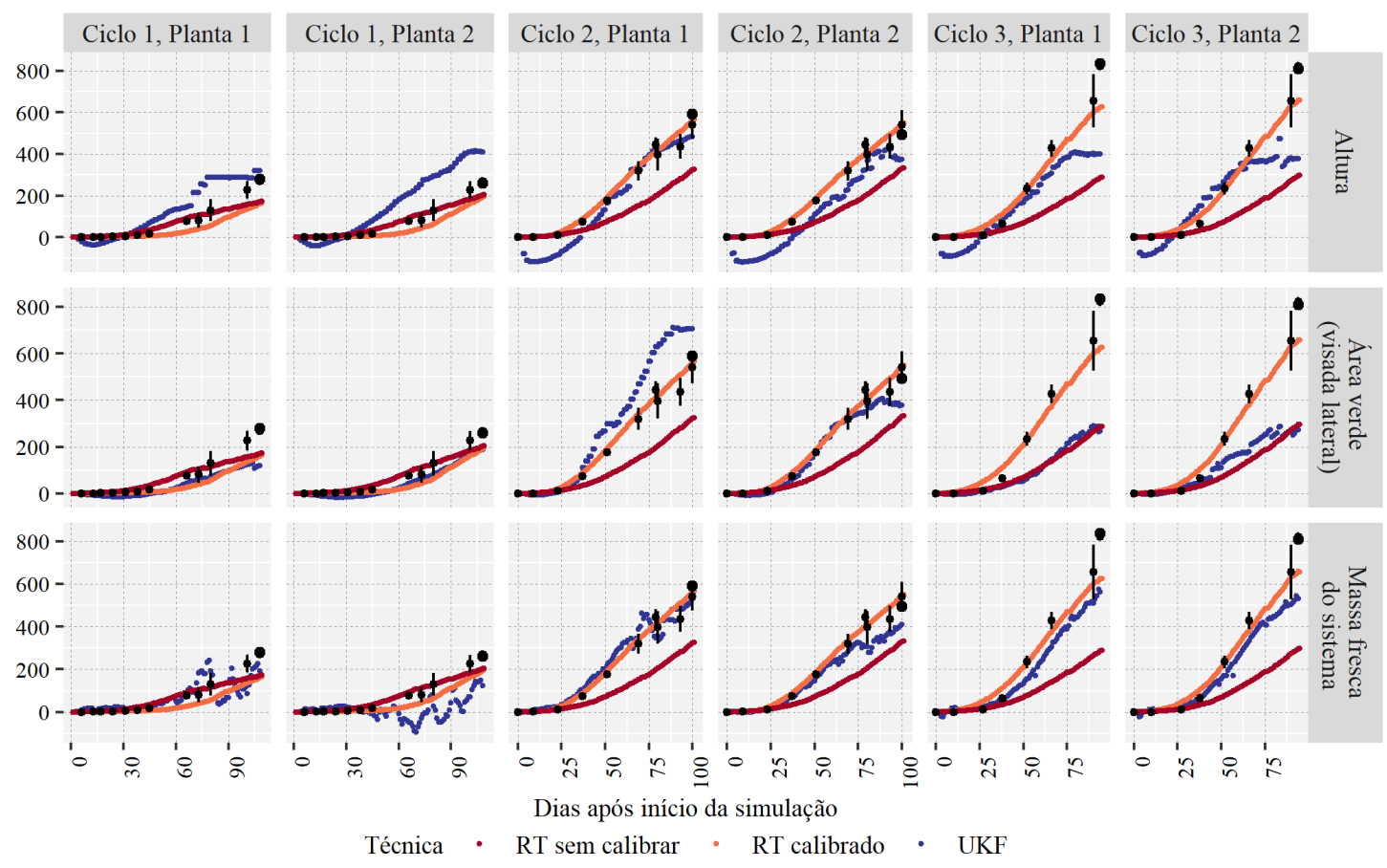

Figura 2. Curvas de crescimento de cada planta monitorada incluindo assimilação utilizando o UKF, simulação calibrada e simulação não calibrada. Os pontos se referem aos valores determinados nas análises destrutivas e a barra representa 0 desvio-padrão dessas amostras. 0 valor final da planta monitorada está representado por um ponto maior.

Um experimento preliminar de assimilação da biomassa seca da parte aérea no RT foi realizado por [Torres-Monsivais et al. 2017]. Embora os autores apresentem poucos detalhes das observações utilizadas, eles concluem que a simulação perturbada com ruídos de até $10 \%$ implica erros de estimativa aceitáveis após a aplicação do UKF. Todavia, os resultados dos autores se referem a uma avaliação em que os erros do modelo são muito baixos por premissa, o que não é necessariamente verdade para modelos não-calibrados. No nosso caso, os erros tanto das observações quanto do modelo atingem magnitudes muito maiores do que os valores simulados, particularmente por serem valores fixos para todo o ciclo. Além disso, a conclusão deles compara erros de magnitudes distintas e, sem uma avaliação dos erros relativos, é possível que ruídos maiores pudessem ser aceitáveis. Sendo assim, as conclusões são 
limitadas e dificultam comparações. A despeito disso, estudos sobre valores adequados para os parâmetros de incerteza são necessários, uma vez que a decisão de como atribuir esses valores de incerteza é um problema em aberto e diversos autores a tomam de formas distintas: alguns autores trabalham com erros variáveis [Lu et al. 2021], outros usam valores fixos também relacionados aos erros do modelo de conversão [Huang et al. 2016], outros estimam com base na variância de observações coletadas em campo [Zhao et al. 2013] enquanto outros usam estratégias que independem dessa incerteza [Chen et al. 2018; Vazifedoust et al. 2009].

Para o Ciclo 03, em que o crescimento se aproximou do potencial, é possível comparar nossos resultados aos obtidos por [Jones et al. 1999]. Os autores também avaliaram o desempenho do modelo sem realizar calibração em duas localidades e concluíram que, embora fosse possível a obtenção de bons resultados para a biomassa, isso não seria possível para a massa de frutos e frutos maduros. Nossos resultados apontaram que a calibração teria sido necessária e que os parâmetros originais implicariam baixa produção de biomassa, possivelmente por incompatibilidade na área foliar, mas a realização de assimilação, permitiu que valores mais próximos aos reais fossem obtidos.

É comum que a assimilação de dados seja considerada uma forma de adequar o crescimento potencial simulado por modelos ao crescimento real. Por exemplo, [Hu et al. 2019] realizaram a assimilação de dados no modelo SWAP-Wofost com o objetivo de contabilizar a interferência no IAF da cana-de-açúcar por remoção de folhas e tempestades naturais. No nosso caso, temos três cenários diferentes: estresse hídrico, estresse nutricional e fertilização e irrigação adequadas. Observamos que o método foi capaz de adaptar a simulação, que espera crescimento potencial, em valores mais realistas ao mesmo tempo em que, para o caso de alta flutuação da água no Ciclo 01, a estimativa foi gravemente deteriorada.

\section{Conclusão}

Nosso trabalho mostra resultados promissores para a assimilação de observações de crescimento por duas perspectivas distintas: ocorre a melhora das estimativas quando não há calibração do modelo e as estimativas obtidas são mais adequadas às condições reais de produção, ao invés da limitação existente na simulação à condição de produção potencial. Também mostramos como observações de diversas fontes, incluindo fotos capturadas por câmeras de baixo custo, foram capazes de caracterizar o crescimento das plantas. Embora nem sempre as relações obtidas tenham sido boas, elas foram suficientemente boas para que informações imperfeitas de observações contínuas pudessem ser usadas juntamente a um modelo imperfeito. Sendo assim, há espaço ainda para que fatores, como o desenvolvimento de modelos de observação com mais dados, sejam mais explorados, de modo que eles possam ser considerados mais generalizáveis e que as análises destrutivas necessárias para a calibração possam ser de fato dispensadas.

\section{Agradecimentos}

O presente trabalho foi realizado com apoio da Coordenação de Aperfeiçoamento de Pessoal de Nível Superior - Brasil (CAPES) - Código de Financiamento 001 e pelo processo $n^{\circ}$ 2018/12050-6, Fundação de Amparo à Pesquisa do Estado de São Paulo (FAPESP). 


\section{Referências bibliográficas}

Bojacá, C. R., Gil, R. and Cooman, A. (mar 2009). Use of geostatistical and crop growth modelling to assess the variability of greenhouse tomato yield caused by spatial temperature variations. Computers and Electronics in Agriculture, v. 65, n. 2, p. 219-227.

Chen, W.-T., Yeh, Y.-H. F., Liu, T.-Y. and Lin, T.-T. (31 mar 2016). An Automated and Continuous Plant Weight Measurement System for Plant Factory. Frontiers in Plant Science, v. 7, n. MAR2016, p. 392.

Chen, Y., Zhang, Z. and Tao, F. (1 nov 2018). Improving regional winter wheat yield estimation through assimilation of phenology and leaf area index from remote sensing data. European Journal of Agronomy, v. 101, p. 163-173.

De Graaf, R., De Gelder, A. and Blok, C. (2004). Advanced weighing equipment for water, crop growth and climate control management. Acta Horticulturae, v. 664, p. $163-167$.

De Koning, A. N. M. and Bakker, J. C. (mar 1992). In situ plant weight measurement of tomato with an electronic force gauge. Acta Horticulturae, n. 304, p. 183-186.

Dorigo, W. A., Zurita-Milla, R., De Wit, A. J. W., et al. (may 2007). A review on reflective remote sensing and data assimilation techniques for enhanced agroecosystem modeling. International Journal of Applied Earth Observation and Geoinformation, v. 9, n. 2, p. 165-193.

Gong, L., Yu, M., Jiang, S., Cutsuridis, V. and Pearson, S. (1 jul 2021). Deep Learning Based Prediction on Greenhouse Crop Yield Combined TCN and RNN. Sensors, v. 21, n. 13, p. 4537.

Helmer, T., Ehret, D. L. and Bittman, S. (2005). CropAssist, an automated system for direct measurement of greenhouse tomato growth and water use. Computers and Electronics in Agriculture, v. 48, n. 3, p. 198-215.

Hemming, S., De Zwart, F. De, Elings, A., Petropoulou, A. and Righini, I. (11 nov 2020). Cherry Tomato Production in Intelligent Greenhouses-Sensors and AI for Control of Climate, Irrigation, Crop Yield, and Quality. Sensors, v. 20, n. 22, p. 6430 .

Hemming, S., De Zwart, F., Elings, A., Righini, I. and Petropoulou, A. (16 apr 2019). Remote Control of Greenhouse Vegetable Production with Artificial IntelligenceGreenhouse Climate, Irrigation, and Crop Production. Sensors, v. 19, n. 8, p. 1807.

Hu, S., Shi, L., Huang, K., et al. (15 feb 2019). Improvement of sugarcane crop simulation by SWAP-WOFOST model via data assimilation. Field Crops Research, v. 232, p. 49-61.

Huang, J., Gómez-Dans, J. L., Huang, H., et al. (15 oct 2019). Assimilation of remote sensing into crop growth models: Current status and perspectives. Agricultural and Forest Meteorology, v. 276-277, p. 107609.

Huang, J., Sedano, F., Huang, Y., et al. (2016). Assimilating a synthetic Kalman filter leaf area index series into the WOFOST model to improve regional winter wheat yield estimation. Agricultural and Forest Meteorology, v. 216, p. 188-202. 
Jin, X., Kumar, L., Li, Z., et al. (jan 2018). A review of data assimilation of remote sensing and crop models. European Journal of Agronomy, v. 92, n. November 2017, p. $141-152$.

Jones, J. W., Kenig, A. and Vallejos, C. E. (1999). Reduced state-variable tomato growth model. Transactions of the ASAE, v. 42, n. 1, p. 255-265.

Keating, B. A. and Thorburn, P. J. (22 oct 2018). Modelling crops and cropping systems - Evolving purpose, practice and prospects. European Journal of Agronomy, v. 100, n. April, p. 163-176.

Lee, J. W. and Son, J. E. (18 oct 2019). Nondestructive and Continuous Fresh Weight Measurements of Bell Peppers Grown in Soilless Culture Systems. Agronomy, v. 9, n. 10, p. 652.

Linker, R. and Ioslovich, I. (2017). Assimilation of canopy cover and biomass measurements in the crop model AquaCrop. Biosystems Engineering, v. 162, p. 5766.

Lu, Y., Chibarabada, T. P., Ziliani, M. G., et al. (2021). Assimilation of soil moisture and canopy cover data improves maize simulation using an under-calibrated crop model. Agricultural Water Management, v. 252, n. April, p. 106884.

Marcelis, L. F. M., Van den Boogaard, R. and Meinen, E. (2000). Control of Crop Growth and Nutrient Supply by the Combined Use of Crop Models and Plant Sensors. IFAC Proceedings Volumes, v. 33, n. 19, p. 161-166.

Pellenq, J. and Boulet, G. (may 2004). A methodology to test the pertinence of remotesensing data assimilation into vegetation models for water and energy exchange at the land surface. Agronomie, v. 24, n. 4, p. 197-204.

Ruíz-García, A., López-Cruz, I. L., Ramírez-Arias, A. and Rico-Garcia, E. (may 2014). Modeling uncertainty of greenhouse crop lettuce growth model using Kalman Filtering. Acta Horticulturae, v. 1037, n. 1037, p. 361-368.

Torres-Monsivais, J. C., López-Cruz, I. L., Ruíz-García, A., Ramírez-Arias, J. A. and Peña-Moreno, R. D. (2017). Data assimilation to improve states estimation of a dynamic greenhouse tomatoes crop growth model. Acta Horticulturae, n. 1170, p. $433-440$.

Vazifedoust, M., Van Dam, J. C., Bastiaanssen, W. G. M. and Feddes, R. A. (2009). Assimilation of satellite data into agrohydrological models to improve crop yield forecasts. International Journal of Remote Sensing, v. 30, n. 10, p. 2523-2545.

Verdouw, C., Tekinerdogan, B., Beulens, A. and Wolfert, S. (1 apr 2021). Digital twins in smart farming. Agricultural Systems, v. 189, n. January, p. 103046.

Yu, D., Zha, Y., Shi, L., et al. (2020). Improvement of sugarcane yield estimation by assimilating UAV-derived plant height observations. European Journal of Agronomy, v. 121, n. February, p. 126159.

Zhao, Y., Chen, S. and Shen, S. (dec 2013). Assimilating remote sensing information with crop model using Ensemble Kalman Filter for improving LAI monitoring and yield estimation. Ecological Modelling, v. 270, p. 30-42. 